REVUE DE L'INSTITUT

FRANÇAIS D'HISTOIRE

EN ALLEMAGNE

\section{Revue de l'IFHA}

Revue de l'Institut français d'histoire en Allemagne

\title{
Une histoire culturelle du pouvoir en Allemagne (Moyen Âge, époque moderne) : autour de Barbara Stollberg-Rilinger
}

Journée d'études, Lyon, 12 février 2010

\section{Guillaume Garner}

\section{OpenEdition}

Journals

Édition électronique

URL : http://journals.openedition.org/ifha/228

DOI : 10.4000/ifha.228

ISSN : 2198-8943

Éditeur

IFRA - Institut franco-allemand (sciences historiques et sociales)

Édition imprimée

Date de publication : 1 janvier 2010

Pagination : 76-78

ISSN : 2190-0078

Référence électronique

Guillaume Garner, «Une histoire culturelle du pouvoir en Allemagne (Moyen Âge, époque moderne) autour de Barbara Stollberg-Rilinger », Revue de l'IFHA [En ligne], 2 | 2010, mis en ligne le 01 février 2013, consulté le 15 septembre 2020. URL : http://journals.openedition.org/ifha/228

Ce document a été généré automatiquement le 15 septembre 2020

(C)IFHA 


\section{Une histoire culturelle du pouvoir en Allemagne (Moyen Âge, époque moderne) : autour de Barbara Stollberg-Rilinger}

Journée d'études, Lyon, 12 février 2010

\section{Guillaume Garner}

\section{NOTE DE L'ÉDITEUR}

Rapport établi par Guillaume Garner

Le 12 février 2010 s'est tenue à l'ENS Lyon une journée d'études conjointement organisée par l'IFHA, l'ENS Lyon et le SFB 596 de l'Université de Münster sur les renouvellements de l'histoire politique de l'Allemagne au Moyen Âge et à l'époque moderne. L'occasion en était la parution de deux ouvrages rédigés par Barbara Stollberg-Rilinger sur ce thème ${ }^{1}$, et dont l'importance a été reconnue en Allemagne : une synthèse sur l'histoire du Saint-Empire de 1495 à 1806, et le catalogue d'une exposition consacrée aux rituels politiques de 800 à 1800. Le principe de cette manifestation consistait à présenter les positions de B. STOLLBERG-RILINGER, celles-ci étant ensuite commentées et discutées par deux historiens français.

Comme l'a rappelé Guillaume Garner dans son introduction, le Saint-Empire a connu depuis une vingtaine d'années un net regain d'actualité historiographique, et l'originalité des travaux de B. STOLLBERG-RILINGER est d'éclairer la logique du fonctionnement de l'Empire en adoptant une approche précise : celle de l'histoire culturelle du politique, le terme de «culturel» renvoyant non à un objet, mais à une méthode, même si ce programme de recherche est à bien des égards aussi une histoire sociale des pratiques politiques. 
3 B. STOLLBERG-RILINGER souligna d'abord que les rituels, à toutes les époques, ont pour fonction de conférer au pouvoir la visibilité dont il a besoin. Par rituel, elle entend une séquence d'actions d'une certaine durée, qui se répètent d'une manière déterminée et solennelle. Ces rituels ont une dimension performative dans la mesure où ils accomplissent ce qu'ils représentent. Les rituels d'investiture sont un exemple parlant de ces significations, et B. Stollberg-Rilinger insista sur le fait que le rituel ne peut déployer ses effets que si les acteurs y assistent personnellement, la présence personnelle fondant une obligation collective réciproque.

4 Il existe un certain nombre de points communs aux rituels à toutes les époques : ceux-ci mobilisent des «vocables de base" qui se combinent dans des grammaires correspondantes. Mais au-delà de ces permanences, quatre éléments distinguent les rituels des périodes médiévale et moderne de ceux du temps présent : l'importance de la présence personnelle des acteurs, l'exhibition de la richesse des participants qui fait du rituel un miroir de la hiérarchie sociale, l'imbrication du religieux et du politique, et enfin le caractère très ouvert des actes rituels qui peuvent recevoir des interprétations variées.

5 B. Stollberg-Rilinger termina son intervention en soulignant deux aspects. D'une part, ces éléments ont fondé l'efficacité des rituels du Saint-Empire à l'époque moderne: l'Empire existait principalement dans ces rituels qui le légitimaient aux yeux des contemporains. D'autre part, cette efficacité s'est érodée à partir de 1648 et des traités de Westphalie, qui ont rendu de plus en plus superflue la présence personnelle des princes d'Empire aux rituels, ce qui avait pour effet de les dégager - au moins en partie - des obligations mises en scène par ces rituels. C'est cette perte de sens que les événements de la période révolutionnaire et impériale ont sanctionnée.

6 Christophe Duhamelle (EHESS) s'attacha à replacer ces apports historiographiques dans le contexte du renouveau de l'histoire du Saint-Empire. Celui-ci est lié à l'affirmation de la période moderne (Frühe Neuzeit) comme période à part entière, à une rupture par rapport à une histoire politique traditionnelle longtemps centrée sur la Prusse et au primat des notions de nation et d'État. C'est dire que l'histoire du Saint-Empire a, dans un premier temps, produit un renouveau qui a plus touché les objets (la réhabilitation des institutions impériales) que les méthodes de la recherche, comme en témoignent les relations distantes que cette histoire a longtemps entretenues avec des courants novateurs tels que l'Alltagsgeschichte ou le paradigme de la confessionnalisation. Depuis une dizaine d'années cependant - et les deux ouvrages ici discutés en témoignent - les approches ont également été renouvelées, par l'intégration de la dimension impériale dans de nouveaux programmes de recherche : l'histoire de la communication, le fait confessionnel ou les relations entre gouvernants et gouvernés. En ce sens, le programme d'histoire culturelle du politique est porteur d'un décloisonnement de l'histoire du Saint-Empire qu'il convient de saluer.

7 Après ces propos centrés sur l'historiographie de l'Empire, Naïma Ghermani (Université Grenoble II) s'attacha à la question des rituels dans une perspective d'histoire culturelle du politique. Elle s'attacha pour cela à la difficulté qu'éprouvent les rois et les princes luthériens face aux sacres et notamment au couronnement qui sont fortement marqués par l'empreinte de la théologie catholique. Comme il était exclu d'abandonner tout rituel et tout cérémoniel, la question qui se posait était celle des moyens de légitimer ces nouveaux pouvoirs, tout en se démarquant des princes catholiques. Le débat fut posé en ces termes au moment du couronnement de Christian I ${ }^{\text {er }}$ du Danemark en 1537. 
Comme le montre l'argumentaire développé par les théologiens réformateurs, il s'agissait de rappeler les origines vétérotestamentaires du couronnement, et en même temps de souligner que le couronnement et l'onction perdent leur force sacrale pour être redéfinis comme des usages, des signes inscrivant dans les mémoires l'existence d'une souveraineté voulue par Dieu. Ces discussions confirment donc le rôle central des rituels dans la légitimation du pouvoir politique.

Ce trois interventions ont été suivies d'une discussion fournie au cours de laquelle furent évoquées des questions aussi différentes que celles de l'articulation de la culture de la présence dans les rituels et de la diffusion de l'imprimé, du traitement réservé aux rituels de l'Empire à l'époque du romantisme, des rituels comme illustration d'une culture politique et juridique de la suspension et de la non-décision, ou des apports de la sociologie de Niklas Luhmann à l'étude des sociétés modernes.

\section{NOTES}

1. Barbara Stollberg-Rilinger, Des Kaisers alte Kleider. Verfassungsgeschichte und Symbolsprache des Alten Reiches, München : Beck, 2008 ; Barbara Stollberg-Rilinger, Matthias Puhle, Jutta Götzmann, Gerd Althoff (dir.), Spektakel der Macht. Rituale im Alten Europa 800-1800 (catalogue de l'exposition au Kulturhistorisches Museum de Magdebourg, 21 septembre 2008-4 janvier 2009), Darmstadt: Primus, 2008. Pour un compte rendu de ces deux ouvrages, voir RevueIFHA, 1, 2009, p. 327-329. 